MSM occurred in inner metropolitan Melbourne suburbs, while the cases in heterosexuals occurred in outer Melbourne suburbs.

Conclusion Notified cases of syphilis infection had significantly increased across all population groups but particularly in heterosexual males and females. Campaigns and control measures should be specific for each population group with targeted screening and education in areas with a high number of syphilis cases.

\section{P088 AUSTRALIAN GENERAL PRACTITIONERS' CONSIDERATION OF PELVIC INFLAMMATORY DISEASE IN WOMEN DIAGNOSED WITH AN STI, AND BARRIERS TO PROVIDING PELVIC EXAMINATIONS}

${ }^{1} \mathrm{H}$ Bittleston*, ${ }^{1} \mathrm{~J}$ Coombe, ${ }^{2} \mathrm{M}$ Temple-Smith, ${ }^{3} \mathrm{D}$ Bateson, ${ }^{4} \mathrm{~J}$ Hunady, ${ }^{2} \mathrm{~L}$ Sanci, ${ }^{1} \mathrm{~J}$ Hocking, 1J Goller. 'Melbourne School of Population and Global Health, University of Melbourne, Melbourne, Australia; ${ }^{2}$ Department of General Practice, University of Melbourne, Melbourne, Australia; ${ }^{3}$ Family Planning NSW, Ashfield, Australia; ${ }^{4}$ True Relationships and Reproductive Health, Windsor, Australia

10.1136/sextrans-2021-sti.218

Background Pelvic inflammatory disease (PID) comprises a range of inflammatory disorders of the female upper genital tract, often occurring after a sexually transmissible infection (STI). When left untreated, PID can cause reproductive complications including ectopic pregnancy and infertility. PID is under-diagnosed globally and requires a clinical diagnosis. Speculum and bimanual pelvic examinations are recommended to support a diagnosis.

Methods In 2019, an online survey about chlamydia, including PID diagnosis and management, was distributed to Australianbased general practitioners (GPs). From 323 respondents, $85.8 \%(\mathrm{n}=277)$ responded to multiple-choice questions about PID and $74.6 \%(n=241)$ responded to a free-text question regarding barriers to performing pelvic examinations. We used multivariable logistic regression to analyse factors associated with the frequency GPs conducted pelvic examinations for women reporting symptoms of PID. Qualitative free-text data were explored using thematic analysis.

Results Most GPs routinely ask female patients with an STI about symptoms suggestive of PID, including pelvic pain (86.2\%), abnormal vaginal discharge (95.3\%), abnormal vaginal bleeding (89.5\%), and dyspareunia (79.6\%). Over half reported routinely conducting speculum (69.0\%) and bimanual pelvic $(55.3 \%)$ examinations for women reporting pelvic pain or dyspareunia. Female GPs were more likely to routinely perform speculum (adjusted odds ratio (AOR) 4.6; 95\%CI: 2.6-8.2) and bimanual pelvic examinations (AOR 3.7; 95\%CI: 2.1-6.5). GPs with additional sexual health training were more likely to routinely perform speculum (AOR 2.2; 95\%CI: 1.1-4.2) and bimanual pelvic examinations (AOR 2.1; 95\%CI: 1.2-3.7). Barriers to pelvic examinations included patient reluctance, GP gender, patient health concerns, time pressure, and GP hesitancy to perform an examination due to inexperience and/or uncertainty that it would add to their assessment.

Conclusions Encouragingly, many GPs routinely asked patients diagnosed with an STI about PID symptoms. However, many GPs in this study did not consistently perform pelvic examinations to support a diagnosis, potentially reducing capacity to diagnose PID.
P089 ACCURACY OF INTERPRETATION AND HOME TEST KIT RESULT REPORTING FOR SCREENING OF HUMAN IMMUNODEFICIENCY VIRUS INFECTION

${ }^{1} \mathrm{~T}$ Doan*, ${ }^{1} \mathrm{C}$ Stafylis, ${ }^{1} \mathrm{Q}$ Wang, ${ }^{1} \mathrm{G}$ Vavala, ${ }^{2} \mathrm{~S}$ Lemley, ${ }^{2} \mathrm{~B}$ McLeman, ${ }^{3,4} \mathrm{~S}$ Young, ${ }^{1} \mathrm{H}$ Xie, ${ }^{5} \mathrm{~L}$ Idelson, ${ }^{2} \mathrm{~L}$ Marsch, ${ }^{1} \mathrm{~J}$ Klausner, ${ }^{6}$ NIDA CTN0083 Team. ${ }^{1}$ David Geffen School of Medicine, University of California, Los Angeles, Los Angeles, USA; ${ }^{2}$ Geisel School of Medicine, Dartmouth College, Lebanon, USA; ${ }^{3}$ Department of Emergency Medicine, School of Medicine, University of California, Irvine, Irvine, USA; ${ }^{4}$ Department of Informatics, Bren School of Information and Computer Sciences, University of California, Irvine, Irvine, USA ${ }^{5}$ ETR Associates (Education, Training and Research), Oakland, USA; ${ }^{6}$ National Institute on Drug Abuse, Bethesda, USA

\subsection{6/sextrans-2021-sti.219}

Background Men who have sex with men (MSM) and individuals identifying as Black or Hispanic/Latino experience the largest burden of Human Immunodeficiency Virus (HIV) infection. The OraQuick In-Home HIV Test (OraSure Technologies $($, Pennsylvania, USA) is the only HIV self-test approved by the US Food and Drug Administration. Self-testing can supplement HIV prevention to increase identification of infections among at-risk groups. During a larger study on social media and HIV prevention, we assessed the accuracy of participants' interpretations of their results.

Methods We recruited Black and Latino MSM between 18-30 years through advertisements on internet-based social media, informational sites, and dating sites. Participants ordered a free OraQuick self-test. They tested and interpreted their results following kit instructions. Participants submitted a test kit photograph to a secure online platform to report their results. Two trained researchers reviewed the photographs and interpreted results independently. We calculated the proportion of agreement and kappa coefficient between reviewers and between reviewers and participants.

Results We enrolled 271 participants, 191 (70\%) ordered a kit, 159 (83\%) used it. Of those, 113 (71\%) submitted readable test result images. Among those submitting images, $71.3 \%$ were Black and $30.4 \%$ were Hispanic/Latino, mean age 25 years (SD 3.6). The proportion of agreement in result interpretation between reviewers was 100\% (113/113), kappa coefficient 1.0. The proportion of agreement in result interpretation between participants and reviewers was $97.3 \%$ (110/113). Of the concordant results, 101 (89.4\%) were negative, $7(6.2 \%)$ were positive, $2(1.8 \%)$ were invalid. Of the 3 discordant results, 2 participants interpreted their positive results as invalid and 1 participant interpreted their positive result as negative. The kappa coefficient was 0.85 (95\% CI 0.67-1.0).

Conclusion Most participants submitted results online. The level of agreement of interpretation between participants and researchers was good. Prevention programs could use self-testing during disruptions of care.

\section{P090 CONDYLOMA ACUMINATA IN AN INFANT : CASE REPORT IN A RESOURCE LIMITED SETTING}

${ }^{1} \mathrm{M}$ Enongene Julius*, ${ }^{2}$ Asonganyi, ${ }^{3}$ Tasa, ${ }^{4} \mathrm{O}$ Yong, ${ }^{5} \mathrm{~N}$ Forbinake. ${ }^{1}$ Cameroon Baptist Convention Health Services, Kumba, Cameroon; ${ }^{2}$ Kumba District Hospital, Cameroon, Kumba, Cameroon; ${ }^{3}$ Kumba Baptist Health Center, Cameroon Baptist Convention Health Services, Cameroon, Kumba, Cameroon; ${ }^{4}$ Kumba Baptist Health Center, Cameroon Baptist Convention Health Services, Cameroon, Kumba, Cameroon; ${ }^{5}$ Hebrew University of Jerusalem Braun School of Public Health and Community Medicine, Jerusalem, Israel 
Background Condyloma acuminata are soft, skin colored, fleshy warts that are caused by the Human Papilloma Virus (HPV), typically HPV 6 and 11 genotypes. The disease is highly contagious, can appear singly, confluent, multiple, small or large. Although anogenital warts are considered to be sexually transmitted in adults, this may not be the case for children. Genital warts in children may result from several modes of transmission: from the maternal genital tract autoinoculation, from finger warts and nonsexual transmission from members/careers. Generally diagnosis of anogenital warts is usually made on physical examination. Surgical treatment options include cryotherapy, laser vaporisation, electrocautery and excision. Nonsurgical approaches in children include the use of podophyllotoxin and imiquimod.

Approach We present a 12-month-old female patient presented with a 6 month history of papillomatosis changes in the anogenital area. The child was born by vaginal delivery after full term normal pregnancy. No maternal medical history of genital warts during her pregnancy. She tested negative for Syphilis at pregnancy.

Physical examination of the child was normal except for the presence of multiple light purple skin-colored, confluent verrucous eruptions affecting the vulva and perianal region (Figure 1). Gynecological examination showed no abnormalities. The hymen was intact, and there was no evidence of ulcerations or other signs of trauma to the vaginal or anal orifices. Biopsy of the lesion was not performed. The girl was treated with 25\% Podophyllotoxin solution, carefully applied at the hospital to the lesions once a week and at every visit, there was evident reduction in the number and size of warts. Treatment was continued for a total of 5 weeks, during which time the lesions cleared almost completely.

Conclusion We conclude from this case that condylomas acuminate are not only transmitted sexually but through nonsexual ways as well, such is this case, from the infected mother to the infant. We also report safe and effective treatment of anogenital warts in a child with regular and carefully applied 25\% Podophyllotoxin solution.

\section{P092 HEALTHCARE ACCESS AND SYPHILIS TESTING AMONG BLACK AND WHITE MSM: RESULTS FROM THE NETWORK EPIDEMIOLOGY OF SYPHILIS TRANSMISSION STUDY}

${ }^{1} \mathrm{C}$ Copen*, ${ }^{*} \mathrm{~J}$ Rushmore, ${ }^{2} \mathrm{~J}$ Jennings, ${ }^{3} \mathrm{~W}$ Miller, ${ }^{3} \mathrm{M}$ Spahnie, ${ }^{2} \mathrm{C}$ Tilchin, ${ }^{3} \mathrm{~A}$ Norris Turner, ${ }^{1} \mathrm{R}$ Kirkcaldy, ${ }^{1} \mathrm{~K}$ Bernstein. ${ }^{1}$ Centers For Disease Control and Prevention, Atlanta, USA; ${ }^{2}$ Johns Hopkins University, Baltimore, USA; ${ }^{3}$ The Ohio State University, Columbus, USA

10.1136/sextrans-2021-sti.221

Background In 2018, men who have sex with men (MSM) accounted for nearly $65 \%$ of newly-reported primary and secondary syphilis cases in the U.S. Syphilis rates are higher among Black than White MSM. Barriers to healthcare access among MSM may contribute to racial disparities in syphilis testing which leads to missed diagnoses. These barriers can be exacerbated by geographic differences in healthcare access. We describe differences in the prevalence of syphilis testing by health insurance status comparing Black to White MSM in two cities (Baltimore, $\mathrm{MD}$ and Columbus, $\mathrm{OH}$ ).
Methods The Network Epidemiology of Syphilis Transmission study enrolled 655 sexually active MSM aged $18+$ from January 2019 through March 2020 ( $\mathrm{n}=329$ Black, $\mathrm{n}=209$ White). At the first study visit, participants were asked about current health insurance status (private or public) and syphilis testing in the 12 months prior to enrollment (yes or no). We used chi-square tests to examine health insurance status and syphilis testing by city comparing Black to White MSM.

Results Although not statistically significant, lower percentages of Black than White MSM in Baltimore had health insurance $(79.6 \%$ vs. $92.4 \%, p=0.11)$, while insurance was universally high in Columbus, regardless of race $(95 \%$, $\mathrm{p}=0.09)$. Lower percentages of Black than White MSM in Baltimore had been tested for syphilis in the past 12 months $(61.7 \%$ vs. $78.7 \%, p=0.06)$, but we observed no testing differences by race in Columbus $(65 \%, p=0.99)$. In Baltimore, fewer insured Black MSM had been tested for syphilis in the last 12 months compared to insured White MSM, but the difference was not significant $(63.4 \%$ vs. $78.7 \%, \mathrm{p}=0.12)$ and was similar in Columbus $(64.9 \%$ vs. $66.4 \%, \mathrm{p}=0.91$ ).

Conclusions Consistent, but non-significant, differences in insurance coverage by race in Baltimore compared with Columbus may indicate geographic variation in healthcare access which can impact timely syphilis testing among MSM.

\section{P093 IMPACT OF COVID-19 HARD LOCKDOWN MEASURES ON SEXUAL BEHAVIOUR IN VICTORIA, AUSTRALIA: FINDINGS FROM A NATIONAL ONLINE SURVEY}

'f Coombe*, 'f Goller, ${ }^{1} \mathrm{H}$ Bittleston, ${ }^{1} \mathrm{~F}$ Yuh Shiong Kong, ${ }^{1,2}{ }^{2}$ Williams, ${ }^{3} \mathrm{~J}$ Tomnay, ${ }^{1} \mathrm{~A}$ Vaisey, ${ }^{1,4} \mathrm{~S}$ Malta, ${ }^{5} \mathrm{M}$ Temple-Smith, 'L Bourchier, ${ }^{1} \mathrm{~A}$ Lau, ${ }^{1,2,6} \mathrm{E}$ Chow, ${ }^{1 J} \mathrm{H}$ Hocking. ${ }^{1}$ Melbourne School of Population and Global Health, The University of Melbourne, Melbourne, Australia; ${ }^{2}$ Melbourne Sexual Health Centre, Carlton, Australia; ${ }^{3}$ Centre for Excellence in Rural Sexual Health, Department of Rural Health, The University of Melbourne, Melbourne, Australia; ${ }^{4}$ National Ageing Research Institute Inc, Parkville, Australia; ${ }^{5}$ Department of General Practice, The University of Melbourne, Melbourne, Australia; ${ }^{6}$ Central Clinical School, Monash University, Melbourne, Australia

\subsection{6/sextrans-2021-sti.222}

Background The residents of Victoria (Australia's second most populous state), were subject to a hard lockdown for several months as they experienced a second wave of COVID-19. Victorians could only leave their homes for essential activities, were required to wear facemasks, remain within $5 \mathrm{~km}$ of their homes and were subject to a nightly curfew. Elsewhere in Australia, COVID numbers remained low with no lockdown in place. We examined the impact of the lockdown on the sexual behaviour of Victorians compared with elsewhere in Australia.

Methods Our online survey was open for 2.5 weeks during the lockdown (August 13th to 31st). Participants aged $18+$ were recruited via social media and asked to report on their current sexual practices. Logistic regression was used to calculate the difference in the proportion of practices between Victorians and non-Victorians.

Results 976 people completed the survey: 71\% identified as female, $75 \%$ were aged 18-29 years, 61\% resided in 